\title{
mTOR as a Key Regulator in Maintaining Skeletal Muscle Mass
}

\author{
Mee-Sup Yoon* \\ Department of Molecular Medicine, School of Medicine, Gachon University, Incheon, South Korea
}

Maintenance of skeletal muscle mass is regulated by the balance between anabolic and catabolic processes. Mammalian target of rapamycin (mTOR) is an evolutionarily conserved serine/threonine kinase, and is known to play vital roles in protein synthesis. Recent findings have continued to refine our understanding of the function of mTOR in maintaining skeletal muscle mass. mTOR controls the anabolic and catabolic signaling of skeletal muscle mass, resulting in the modulation of muscle hypertrophy and muscle wastage. This review will highlight the fundamental role of mTOR in skeletal muscle growth by summarizing the phenotype of skeletal-specific mTOR deficiency. In addition, the evidence that $\mathrm{mTOR}$ is a dual regulator of anabolism and catabolism in skeletal muscle mass will be discussed. A full understanding of mTOR signaling in the maintenance of skeletal muscle mass could help to develop mTOR-targeted therapeutics to prevent muscle wasting.

\section{OPEN ACCESS}

Edited by:

Bert Blaauw,

Università degli Studi di Padova, Italy

Reviewed by:

John Joseph McCarthy,

University of Kentucky, United States

Kunihiro Sakuma

Tokyo Institute of Technology, Japan

*Correspondence:

Mee-Sup Yoon

msyoon@gachon.ac.kr

Specialty section:

This article was submitted to

Striated Muscle Physiology,

a section of the journal

Frontiers in Physiology

Received: 20 July 2017 Accepted: 26 September 2017 Published: 17 October 2017

Citation:

Yoon M-S (2017) mTOR as a Key Regulator in Maintaining Skeletal Muscle Mass. Front. Physiol. 8:788.

doi: 10.3389/fphys.2017.00788
Keywords: mTOR, skeletal muscle, hypertrophy, atrophy, sarcopenia

\section{INTRODUCTION}

Skeletal muscle primarily functions as a motor for locomotion, and recently growing evidence has recognized skeletal muscle as a crucial regulator of whole body metabolism (Izumiya et al., 2008; McCarthy and Esser, 2010). Skeletal muscle mass is dependent on diverse conditions, including aging, disuse, cachexia, denervation, and burns (Glass, 2003), and affects disability, loss of independence, and increased risk of morbidity and mortality (Hornberger, 2011). Hence, the maintenance of muscle mass has been recognized as a determinant which directly influences quality of life.

Mechanical overload and anabolic stimulation are suggested to be important for increasing skeletal muscle mass and fiber size. Notably, changes in adult muscle mass by external stimuli emerged from the growth of the individual muscle fibers, not from an increase in the number of muscle fibers (Glass, 2005). One of the most widely recognized major players in controlling muscle mass is mammalian target of rapamycin (mTOR). mTOR is a serine/threonine kinase which senses various environmental and intracellular changes including nutrient availability and energy status, and coordinates diverse cellular processes including cell growth, differentiation, autophagy, survival, and metabolism (Laplante and Sabatini, 2012). Two biochemically and functionally distinct mTOR complexes, mTORC1 and mTORC2, exist (Laplante and Sabatini, 2012). Both complexes have mTOR as their common catalytic subunit and each has unique components. mTORC1 consists of the regulatory associated protein of mTOR (raptor), the $40 \mathrm{kDa}$ prolinerich Akt substrate (PRAS40), the DEP domain-containing mTOR-interacting protein (DEPTOR), and the mammalian lethal with SEC13 protein 8 (mLST8). The other mTOR complex, mTORC2, shares mLST8 and DEPTOR with mTORC1 and includes unique components: the rapamycininsensitive companion of mTOR (rictor), mammalian stress-activated map kinase-interacting 
protein $1(\mathrm{mSIN} 1)$, and the exchange factor found in platelets and leukemia and neuronal tissues (Xpln). The rapamycin sensitive complex mTORC1 integrates several extracellular and intracellular signals including growth factors, amino acid availability, intracellular energy status, and oxygen levels. The tumor suppressor tuberous sclerosis complex TSC1-TSC2 mediates the upstream signals of mTORC1 except for amino acid availability by acting as a GTPase-activating protein (GAP) for the small GTPase Rheb. mTORC1 controls protein synthesis by activating S6 kinase 1 (S6K1) and inhibiting 4Ebinding protein 1 (4EBP1) (Ma and Blenis, 2009). On the other hand, mTORC2 phosphorylates AGC kinases, serum, and glucocorticoid-regulated kinase 1 (SGK1), protein kinase $\mathrm{C}$ (PKC), and Akt, and regulates cell survival and metabolism (Sarbassov et al., 2005).

mTORC1 is known as a key regulator in controlling skeletal muscle mass following contraction and mechanical load-induced hypertrophy, synergistic ablation, myotube hypertrophy, and amino acid sensing, in which mTOR interacts with factors of both skeletal muscle hypertrophy and atrophy. This review explores the critical role of mTORC1 and its signaling in both catabolism and anabolism of skeletal muscle by summarizing genetic and pharmacological evidence and delineating the current understanding of the molecular mechanism of mTOR in the regulation of skeletal muscle mass.

\section{THE PHENOTYPES OF MUSCLE-SPECIFIC DEFICIENCY OF MTOR SIGNALING}

Understanding of the role of mTOR in muscle growth and hypertrophy has progressed recently from the evidence of several loss-of-function animal models, although it is relatively wellunderstood as being similar to the mechanism of the function of mTOR in cell growth regulation. To avoid the early embryonic mortality of mice deficient for mTOR and rictor/raptor, musclespecific knockout mice of mTOR and mTOR components were generated (Guertin et al., 2006; Bentzinger et al., 2008).

Muscle-specific mTOR knockout mice (mTOR-) exhibit severe myopathy leading to premature death between 22 and 38 weeks of age (Risson et al., 2009). Muscle-specific raptor knockout mice (RAmKO) and raptor and rictor doubleknockout mice (DmKO) show myopathy similar to one associated with the loss of mTOR in muscle, whereas musclespecific rictor knockout mice (RImKO) do not. The deficiency of either mTOR or raptor reduces the phosphorylation of mTORC1 downstream targets, such as p70S6K1 and 4EBP1 and increases the phosphorylation of Akt at S473 and T308. Unexpectedly, Akt phosphorylation in DmKO is comparable to phosphorylation in RAmKO, implying that mTORC2 is not required for Akt activation in muscle. In addition, the muscle of RImKO mice is similar to one of wild type controls, suggesting that mTORC1 plays a major role in the metabolic processes and functions of muscle.

Compared to RAmKO/DmKO, mTOR- mice shows changes in muscle contractile properties. Additionally, mTOR- reduces the transcription of dystrophin, resulting in a decrease in the content of the dystrophin-glycoprotein complex (DGC), which connects the cytoskeleton of a muscle fiber to its surrounding extracellular matrix; the disruption of DGC results in muscular dystrophy. mTOR directly binds to the promoter of the dystrophin gene to control the transcription of dystrophin in a cell-autonomous, rapamycin-resistant, and kinase-independent manner. mTOR knockout muscle also undergoes metabolic changes, resulting in glycogen accumulation due to increased glycogen synthesis and glucose uptake together with reduced glycogen breakdown through glycogenolysis and the glycolytic and oxidative pathways. mTORC1 deficiency in muscle significantly reduces the expression of genes in mitochondria biogenesis, such as proliferator-activated receptor $\gamma$ coactivator- 1 alpha (PGC1 $\alpha$ ), myoglobin, PPAR $\gamma$, and cytochrome C oxidase IV (COXIV). However, it does not affect either intramuscular ATP level or whole body glucose homeostasis.

Similar to mTOR deficiency in muscle, RAmKO induces progressive dystrophy, impairment in oxidative capacity, and increased glycogen stores; these mice displayed metabolically fast-twitch, glycolytic skeletal muscle (Bentzinger et al., 2008). This characterization is also reflected in the overall metabolism, which displayed lower glucose uptake from the blood. However, the soleus and EDL of RAmKO muscle had slower myosin heavy chain (slMHC)-positive fibers, indicating that RAmKO muscle contained more structurally slow-twitch, oxidative skeletal muscle fibers. In addition, the deletion of S6K1, an mTORC1 downstream target, in muscle increases AMP/ATP level and activates AMPK, resulting in energy stress and muscle cell atrophy (Aguilar et al., 2007).

Even though the phenotypes of mTORC1-signaling deficient mice are similar in terms of skeletal muscle myopathies, mitochondrial and oxidative metabolism in these mice are distinct. The deficiency of $\mathrm{mTOR}$ and raptor in muscle induces defects in mitochondrial metabolism and a decrease in mitochondrial gene expression (Bentzinger et al., 2008; Risson et al., 2009). This is supported by previous reports that mTORC1 is a positive regulator of $\mathrm{PGC} 1 \alpha$, a master regulator of mitochondrial biogenesis. However, S6K1 -/- skeletal muscle has high mitochondrial content accompanied by increased expression of mitochondrial genes, which protect against dietinduced obesity together with enhanced $\beta$-oxidation in white adipose tissue (WAT) (Um et al., 2004). These observations imply that an mTORC-mediated regulation is vital for mitochondrial metabolism in metabolism-related organ, which is differentially regulated in muscle and WAT, respectively. This differential regulation of mitochondrial metabolism warrants further investigation.

\section{mTOR SIGNALING REGULATES MUSCLE PROTEIN SYNTHESIS}

\section{The Role of mTOR in IGF-I Dependent Pathway of Skeletal Muscle}

Numerous reports show that IGF-I is a requisite for muscle growth and regeneration (Florini et al., 1991; Vandenburgh et al., 1991; Coleman et al., 1995; Musaro et al., 2001; 
Rabinovsky et al., 2003; Pelosi et al., 2007), as well as a wellknown upstream stimulator of mTOR in skeletal muscle. IGFI binds to IGF-I receptor (IGFR), a receptor tyrosine kinase, and subsequently recruits insulin receptor substrate-1 (IRS-1). Although IRS-1 activates the Ras-Raf-MEK-ERK pathway, the role of this pathway in skeletal muscle is not clear (Rommel et al., 1999). Instead, Akt /mTOR signaling by IGF-I/IGFR/IRS1 has been shown to be indispensable in prompting muscle hypertrophy (Glass, 2003). Akt phosphorylates TSC1/2, which inhibits the GTPase-activating protein (GAP) activity of TSC1/2 toward small G protein Rheb. Then, GTP-bound Rheb activates mTORC1, resulting in phosphorylation of S6K1and 4EBP1, which promote protein synthesis by activating ribosomal protein S6 and by releasing the translation initiation factor eIF-4E, respectively. In line with IGF-I-Akt-mTORC1 regulation, IGFI induces hypertrophy of skeletal myofiber in tissue culture (Vandenburgh et al., 1991). Muscle-specific expression of IGFI in transgenic mice results in at least a 2 -fold increase in muscle hypertrophy (Coleman et al., 1995; Musaro et al., 2001), suggesting that the IGF-I/Akt/mTORC1 pathway is indispensable to muscle hypertrophy. In addition, Akt regulates muscle mass by phosphorylating and deactivating glycogen synthase kinase (GSK) $\beta 1$, followed by the GSK $\beta 1$-dependent inhibition of the eukaryotic translation initiation factor 2B (eIF2B) (Manning and Cantley, 2007; Schiaffino and Mammucari, 2011).

However, a recent report showed that IGF-I and its receptor IGFR were not important to the induction of hypertrophy and the activation of Akt/mTOR in mechanical loading (Spangenburg et al., 2008). The expression of dominant negative (DN)IGF-I receptor specifically in skeletal muscle induced muscle hypertrophy using an increased functional overload model induced by synergistic ablation (Spangenburg et al., 2008). Of interest, DN-IGF-I receptor-expressing muscle showed a similar level of activation of Akt and p70S6K1. These results implied that an unknown upstream mediator beyond IGFR might regulate Akt/mTOR signaling in skeletal muscle hypertrophy.

\section{The Role of mTOR in IGF-I Independent Pathway of Skeletal Muscle; PA-Induced mTOR Activation in Mechanical Stimulus}

One of the potential IGFR-independent mTOR regulators in skeletal muscle is phosphatidic acid (PA). Hornberger et al. observed that IGF-I-independent mechanical stretch increases phosphatidic acids (PA), followed by mTOR activation (Hornberger et al., 2006). PA directly binds to the FKBP12rapamycin binding (FRB) domain in competition with rapamycin, and activates mTOR (Fang et al., 2001). PA is synthesized through several pathways: from phosphatidylcholine (PC) by phospholipase D (PLD), from lysophosphatidic acid (LPA) by lysophosphatidic acid acyltransferases (LPAAT), and from diacylglycerol (DAG) by diacylglycerol kinase (DGK) (Wang et al., 2006; Yoon et al., 2015). Among the several enzymes involved in PA biogenesis, PLD activity was increased by mechanical stretch and followed by mTOR activation (Hornberger et al., 2006). In addition, treatment with 1-butanol, a PLD inhibitor, inhibited the increase in mTOR activity, supporting the role of PLD in mechanical stretch (Hornberger et al., 2006). However, PA level continued to remain high after the elevated PLD activity returned to basal level $15 \mathrm{~min}$ after mechanical stretch using $[3 \mathrm{H}]$ arachidonic acid labeling, suggesting that other enzymes produce PA under mechanical stretch. Hornberger et al. found that DGK $\zeta$ produces PA under mechanical stimulation, which is followed by mTOR activation (You et al., 2014). Mechanical stimulation does not induce PLD activity under $[3 \mathrm{H}]$ myristic acid labeling that preferentially labels PC, and FIPI, a PLD inhibitor, did not inhibit mechanical stimulation. Instead, both DAG and membrane DGK activity, which are critical for mTOR activation, were increased during mechanical stimulation. However, the previous report suggested that PLD1-produced PAs preferentially bind to the FRB domain of mTOR and displace DEPTOR, leading to mTORC1 activation (Yoon et al., 2015). Hence, further investigation into whether PA produced by DGK $\zeta$ during muscle stretch binds to the FRB domain or activates mTOR through an FRB-independent mechanism is warranted.

\section{The Role of mTOR in IGF-I Independent Pathway of Skeletal Muscle; Mechanism of mTORC1 Activation by Mechanical Stimulus}

It has been established that mTORC1 translocates to the lysosome through regulation of Ragulator-Rag in amino acid signaling (Sancak et al., 2008, 2010). Lysosomal localization of mTOR does not activate mTOR directly, but rather provides close proximity to Rheb, an essential activator of mTOR (Saxton and Sabatini, 2017). mTORC1 is activated by direct interaction with the GTP-bound form of Rheb (Sancak et al., 2008, 2010), which is regulated by the TSC complex [TSC1, TSC2, and Tre2Bub2-Cdc16-1 domain family member 7 (TBC1D7) Dibble and Manning, 2013], a GAP of Rheb (Huang and Manning, 2008; Saxton and Sabatini, 2017). The location of Rheb, shown to be on the lysosome, was not changed by either amino acids or insulin (Menon et al., 2014). Nevertheless, the TSC complex activates the intrinsic GTPase activity of Rheb on the surface of the lysosome and localizes to the lysosome, at least partially through its association with Rheb-GDP in the absence of growth factors (Menon et al., 2014). Insulin activates Akt, which subsequently phosphorylates the TSC complex, resulting in the dissociation of the TSC complex from Rheb, followed by Rheb GTP loading and mTORC1 activation (Menon et al., 2014).

The Hornberger group found that mTOR and TSC2 were highly enriched in the lysosome of the muscle in the resting state (Jacobs et al., 2013). Mechanical stimulation induces TSC2 phosphorylation at $\mathrm{RxRxx}^{*} / \mathrm{T}^{*}$, which resulted in the dissociation of TSC2 from the lysosome and the subsequent change of Rheb to the active Rheb-GTP state. Furthermore, mechanical stimulation also facilitates the association of mTOR with the lysosome. Accordingly, mTOR potentiates the activation of the lysosome through the interactions with Rheb-GTP or PA, as previously reported (Sancak et al., 2008, 2010; Yoon et al., 2011). However, the kinase for TSC phosphorylation was unclear in this study since mechanical stimulation was 
previously shown to activate mTOR in PI3K/Akt-independent manner (Hornberger et al., 2004; O’Neil et al., 2009). In addition, Song et al. recently suggested the colocalization of mTOR with eukaryotic translation initiation factor 3 subunit $F$ (eIF3F) in resistance exercise (Song et al., 2017). mTOR is localized on the lysosome in the basal state and the mTOR-LAMP2 complex is translocated to the cell periphery under resistance exercise, which provide close proximity to the capillaries. In support of this, the lysosome is shown to migrate to the cell periphery after nutrient stimulation through two kinetin proteins, K1F1B $\beta$ and KIF2, which are essential to mTORC1 activation (Korolchuk et al., 2011). Concurrently, TSC2 dissociates from Rheb, followed by the reduction of TSC2 on the cell periphery and the subsequent increase of mTORC1 activity (Song et al., 2017). In addition, both the association of mTOR with eIF3F and S6K1 activity are increased in fed conditions after exercise, which provides an explanation for the enhanced muscle protein synthesis (Song et al., 2017). Nevertheless, the association of mTOR with eIF3F was mainly determined by using an immunofluorescent approach in Song's study, which requires further investigation by using a range of techniques.

\section{mTOR REGULATION SIGNALS MUSCLE WASTING}

The loss of skeletal muscle, muscle atrophy, stems from an increase in the rate of protein degradation or the decrease of protein synthesis under various conditions, such as disuse, diseases, and aging. In line with mTOR function as a positive regulator of muscle hypertrophy, mTOR signaling is negatively regulated by muscle atrophy-inducing signals or blocks muscle atrophy signals. In this section, it will be discussed the crosstalk between mTOR and two major muscle atrophy-inducing signals such as myostatin and glucocorticoids.

\section{The Crosstalk between mTOR and Myostatin (Growth Differentiation Factor 8, GDF-8)}

Myostatin, a transforming growth factor- $\beta$ (TGF- $\beta$ ) family member, plays a critical role in inhibiting the growth of muscle mass and muscle cell differentiation (McPherron et al., 1997). The deletion of myostatin in mice results in muscle hyperplasia and hypertrophy, and more than doubles skeletal muscle (McPherron et al., 1997). Myostatin regulates the number of muscle fibers during development and the growth of muscle fibers postnatally (Lee, 2007). The binding of myostatin to the type II activin receptor IIb leads to interaction with the type I receptor ALK4 or ALK5, which results in the phosphorylation and activation of the transcription factors Smad2 and Smad3(Sartori et al., 2014). Additionally, myostatin decreases Akt phosphorylation, which is accompanied by the accumulation of dephosphorylated active Forkhead Box-O1 (FOXO1) and FOXO3, followed by upregulation of components of the ubiquitin-proteasome pathway, such as atrogin-1 and the muscle-specific E3 ubiquitin ligase muscle RING-finger1 (MURF1) (McFarlane et al., 2006; Lokireddy et al., 2011). In addition, myostatin blocks differentiation-inducing genes, such as myogenin and myoD (Trendelenburg et al., 2009), suggesting that myostatin regulates muscle differentiation by modulating both the programs of differentiation and atrophy.

mTOR regulation by myostatin has sophisticated the molecular mechanism of myostatin signaling. The overexpression of myostatin decreases Akt and mTORC1 components, such as p70S6K1, S6, and 4EBP1 (Amirouche et al., 2009). Supporting the negative regulation of myostatin in mTORC1 signaling, genetic deletion of myostatin elevates the activities and the expression levels of Akt, p70S6K1, and S6 (Lipina et al., 2010). Additionally, treatment with myostatin reduces myoblast differentiation and myotube size by inhibiting the activity of Akt/mTORC1/p70S6K1 in human skeletal muscle cells (HuSkMC) (Trendelenburg et al., 2009). The depletion of raptor increases myostatin-induced Smad2 phosphorylation, followed by further inhibition of myostatin-induced muscle differentiation. The knockdown of rictor itself inhibits muscle cell differentiation, and does not affect myostatin-induced pSmad2 and muscle differentiation. These results suggested that both mTORC1 and myostatin-Smad2 signaling negatively regulate each other. The overexpression of ActRIIB induces inhibition of myostatin, resulting in skeletal muscle hypertrophy, which is reduced partially by treatment with rapamycin (Sartori et al., 2009). Hence, the studies suggested that myostatin attenuates protein synthesis in muscle by coordinating the crosstalk between myostatin-mediated and mTOR signaling. However, on the other hand, several studies suggested that mTOR signaling and myostatin signaling could separately regulate muscle growth. The injection of a myostatin antibody enhances phosphorylation of p70S6K1 and S6 in muscle, but does not change phosphorylation of Akt and 4EBP1 in the concomitant increase of myofibrillar synthesis (Welle et al., 2009). In this study, treatment with rapamycin does not affect myofibrillar synthesis, while it decreases the phosphorylation of p70S6K1 and S6, implying that mTOR is not involved in myostatin-mediated myofibrillar synthesis (Welle et al., 2009). In addition, follistatin, an inhibitor of myostatin, activates Akt $/ \mathrm{mTOR} / \mathrm{p} 70 \mathrm{~S} 6 \mathrm{~K} 1 / \mathrm{S} 6$ signaling in muscle growth, which exists independently of myostatin-driven mechanisms (Winbanks et al., 2012), supporting the disconnection between myostatin and mTOR signaling. Hence, myostatin may regulate protein synthesis in both an mTOR-dependent and an mTORindependent manner; it controls the translation through Akt/mTORC1/p70S6K1/S6 signaling and, at the same time, it directly acts on unknown regulators of translation.

\section{The Role of mTOR in Glucocorticoid-Induced Atrophy}

Glucocorticoids are some of the most fundamental regulators of energy homeostasis and adjust the metabolism of carbohydrates, fat, and protein in skeletal muscle (Munck et al., 1984). Glucocorticoids bind to the glucocorticoid receptor (GR), which translocates to the nucleus and binds to the glucocorticoid response element (GRE) in the promoters of target genes (Meijsing et al., 2009). Notably, the circulating levels of 
glucocorticoids are increased under many pathological conditions which are accompanied by muscle atrophy such as cachexia, starvation, sepsis, metabolic acidosis, and severe insulinopenia (Braun and Marks, 2015). Exogenous administration of glucocorticoids induces muscle atrophy and the blockage of GR; adrenalectomy or treatment with the GR antagonist RU486 diminishes muscle atrophy in sepsis, cachexia, starvation, and severe insulinopenia (Menconi et al., 2007; Schakman et al., 2008). Hence, endogenous glucocorticoids are critical regulators in muscle atrophy.

The crosstalk between GR and mTOR has been reported in muscle cells (Shimizu et al., 2011). Tanaka et al. found that REDD1 and KLF15 inhibit mTOR activation as direct targets of GR. REDD1 has a functional GRE, which sequestrates 143-3 from TSC1/2, resulting in activation of mTOR (DeYoung et al., 2008). KlF15 plays a critical role in muscle catabolism through the transcriptional upregulation of atrogen-1, MuRF1 , and branched-chain aminotransferase 2 (BCAT2). BCAT2 catalyzes the first reaction of BCAA catabolism, facilitating BCAA degradation, followed by mTOR inactivation and decrease of myofiber size (Shimizu et al., 2011). On the other hand, mTOR negatively modulates GR-mediated transcription by inhibiting GR recruitment of target genes. Hence, both GR and mTOR control each other exclusively in the regulation of muscle mass.

Glucocorticoids also elicit muscle atrophy via controlling transcription of myostatin, an inhibitory regulator of muscle growth, which we discussed in the previous section. Human myostatin promoter is reported to have a putative GRE and is responsive to dexamethasone and RU-486, an antagonist of GR (Ma et al., 2001). Indeed, the expression of myostatin
mRNA and protein are increased in a dose-dependent manner in dexamethasone-treated rats (Ma et al., 2003). Myostatin production is also induced by food deprivation in a glucocorticoid-dependent manner (Allen et al., 2010). A recent report also suggests that glucocorticoids increase phosphorylation of CEBP by decreasing PDE3/4 and activating PKA through inhibiting Akt activity, resulting in increased myostatin expression (Xie et al., 2017). Hence, glucocorticoids may regulate mTOR by modulating the level of both BCAT2 and myostatin to regulate catabolism in skeletal muscle.

\section{mTOR AND SARCOPENIA}

Sarcopenia has been defined as an age-related continuous decline in muscle mass, quality, and strength (Sakuma et al., 2014). It is characterized by overall decreases in size and number of skeletal muscle fibers, mostly the type 2 or fast-twitch muscle fibers, and a marked infiltration of fibrous and adipose tissue into the skeletal muscle (Walston, 2012). The amount of circulatory IGFI and IGF-I mRNA levels are reduced (Leger et al., 2008), and subsequently the activity of Akt/mTOR/p70S6K1 are decreased in older age groups compared to one in younger groups (Pallafacchina et al., 2002; Cuthbertson et al., 2005; Leger et al., 2008). A study using inducible liver IGF-I-deficient mice revealed that reduced IGF-I at 1 year of age is related to deteriorated health accompanied by age-related pathologies (Gong et al., 2014), consistent with the previous report that virus-mediated IGF-I gene transfer counteracts the decreases of muscle mass and force in aged groups(Barton-Davis et al., 1998). In addition, the levels of IGF-binding proteins in aged groups increased, followed by

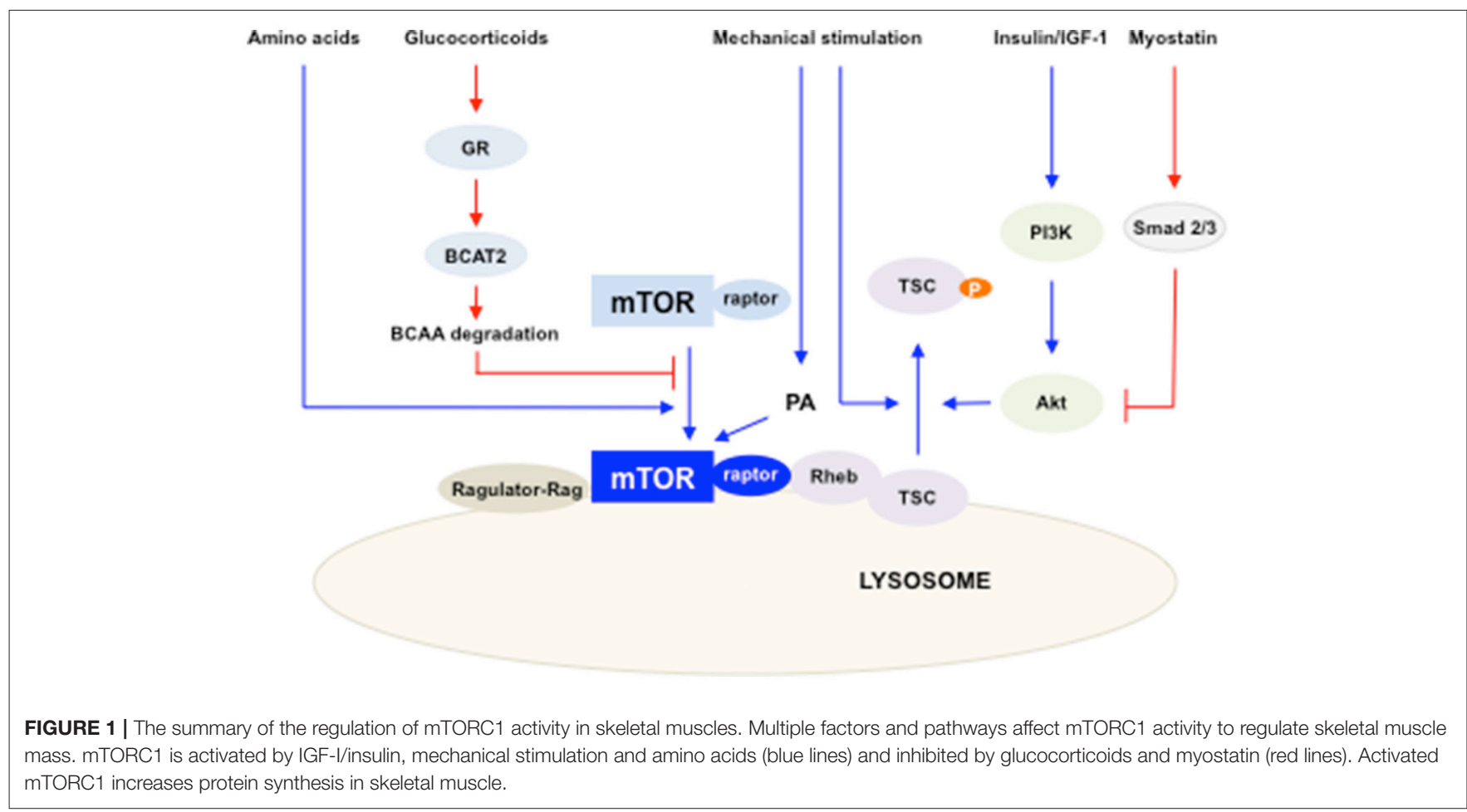


inactivation of Akt, impaired differentiation, and hypertrophy of myotubes (Deane et al., 2013; Sharples et al., 2013), suggesting that lowering of IGF-I signaling are responsible for the decrease of skeletal muscle mass in old-aged group. On the other hand, recent papers report contradicting results in IGF-I levels and activity of Akt/mTOR/p70S6K1 in aged muscles. IGF-1 mRNA expression was not changed or even increased in aged muscles compared to young muscles in humans (Sandri et al., 2013), whereas it was decreased in aged muscle of mice (Drummond et al., 2008; Sandri et al., 2013). Moreover, the correlation between IGF-1 level and Akt/mTOR was not consistent in several reports (Sandri et al., 2013; Markofski et al., 2015). Thus, the attempt to change IGF-I levels in aged muscle for sarcopenia warrants further investigations

Of note, the hyperphosphorylation of mTORC1 was observed in aged human muscles (Sandri et al., 2013; Markofski et al., 2015). Inhibition of mTORC1 has a positive effect in the animal model of age-associated muscular dystrophy (Ramos et al., 2012). Related to this, reduced mTOR signaling has been shown to regulate longevity in human and model organisms (Powers et al., 2006; Bjedov et al., 2010; Robida-Stubbs et al., 2012; Passtoors et al., 2013) and reduce age-related pathologies (Johnson et al., 2013a). Inhibition of mTOR signaling in aged muscle may have similar beneficial effects on multiple age related pathologies (Johnson et al., 2013b). Nevertheless, the hyperactivation of mTOR in aged muscles does not induce protein synthesis (Markofski et al., 2015). Chronic mTORC1 activation through TSC1 knockout in old muscle leads to muscle atrophy mainly due to inability to induce autophagy (Castets et al., 2013), suggesting the importance of mTOR-induced regulation of autophagy in aged muscle. Moreover, the hyperphosphorylation of mTOR might lead to resistance to anabolic stimuli in aged muscle. Anabolic stimuli such as muscle contraction, insulin, and nutrients cause an increase in protein synthesis through mTOR activation in the muscle, and this anabolic stimuliinduced mTOR activation is reduced in individuals belonging to older age groups compared to younger adults (Parkington et al., 1985; Cuthbertson et al., 2005; Chale-Rush et al., 2009; Timmerman et al., 2010; Fry et al., 2011; Li et al., 2012). Hence, the hypertrophic response by mTOR activation is important for overall muscle maintenance in aged muscle.

\section{CONCLUSIONS AND PERSPECTIVES}

Our understating of mTOR regulation in both skeletal muscle hypertrophy and atrophy has been advanced in recent years.

\section{REFERENCES}

Aguilar, V., Alliouachene, S., Sotiropoulos, A., Sobering, A., Athea, Y., Djouadi, F., et al. (2007). S6 kinase deletion suppresses muscle growth adaptations to nutrient availability by activating AMP kinase. Cell Metab. 5, 476-487. doi: 10.1016/j.cmet.2007.05.006

Allen, D. L., Cleary, A. S., Hanson, A. M., Lindsay, S. F., and Reed, J. M. (2010). CCAAT/enhancer binding protein- $\delta$ expression is increased in fast skeletal muscle by food deprivation and regulates myostatin transcription
While mTOR has been appreciated as a main regulator of protein synthesis in skeletal muscle, its crosstalk with muscle atrophy inducing triggers, such as myostatin and glucocorticoids, have been studied. The diverse involvements of mTOR in maintaining skeletal muscle mass have shed light on the complexity of the role of mTOR in skeletal muscle hypertrophy and atrophy (Figure 1). Hence, dissection of mTOR signaling provides useful potential therapeutic strategies in boosting skeletal muscle growth and preventing muscle loss.

Recent studies suggest an additional role of mTOR in skeletal muscle related to the regulation of non-coding RNAs. MicroRNAs (miRNAs) are small non-coding RNAs that are 21-23 nucleotides in length, which bind to $3^{\prime}$-UTR of target mRNAs and function in gene silencing and translational suppression (Zhang et al., 2016). Several miRNAs are identified as myomiRNAs, which are enriched in skeletal muscle and known to modulate the cellular processes involved in muscle growth, development, and maintenance, including hypertrophy and atrophy. The expression of several miRNAs, such as miR1, miR-133, miR-206, and miR-125b, are regulated by mTOR directly or indirectly (Sun et al., 2010; Ge et al., 2011), suggesting the additional regulation of $\mathrm{mTOR}$ in skeletal muscle mass. Recently, a novel polypeptide encoded by the long non-coding RNA (lncRNA) LINC00961 was shown to regulate mTOR activation and muscle regeneration (Matsumoto et al., 2017), implying crosstalk between mTOR and non-coding RNAs in skeletal muscle. In this context, future studies of mTOR signaling as a possible therapeutic target using non-coding RNAs are warranted.

\section{AUTHOR CONTRIBUTION}

The author confirms being the sole contributor of this work and approved it for publication.

\section{FUNDING}

This research was supported by the Basic Science Research Program through the National Research Foundation of Korea (NRF) and funded by the Ministry of Education (Grant number: NRF-2015R1D1A1A01058313), the Korea Health technology R\&D Project through the Korea Health Industry Development Institute (KHIDI), funded by the Ministry of Health \& Welfare (Grant number: HI17C0426) and the Gachon University Gil Medical Center (Grant number: 2015-15). in vitro. Am. J. Physiol. Regul. Integr. Compar. Physiol. 299, R1592-R1601. doi: 10.1152/ajpregu.00247.2010

Amirouche, A., Durieux, A. C., Banzet, S., Koulmann, N., Bonnefoy, R., Mouret, C., et al. (2009). Down-regulation of Akt/mammalian target of rapamycin signaling pathway in response to myostatin overexpression in skeletal muscle. Endocrinology 150, 286-294. doi: 10.1210/en.2008 $-0959$

Barton-Davis, E. R., Shoturma, D. I., Musaro, A., Rosenthal, N., and Sweeney, H. L. (1998). Viral mediated expression of insulin-like growth factor I blocks the 
aging-related loss of skeletal muscle function. Proc. Natl. Acad. Sci. U.S.A. 95, 15603-15607. doi: 10.1073/pnas.95.26.15603

Bentzinger, C. F., Romanino, K., Cloetta, D., Lin, S., Mascarenhas, J. B., Oliveri, F., et al. (2008). Skeletal muscle-specific ablation of raptor, but not of rictor, causes metabolic changes and results in muscle dystrophy. Cell Metab. 8, 411-424. doi: 10.1016/j.cmet.2008.10.002

Bjedov, I., Toivonen, J. M., Kerr, F., Slack, C., Jacobson, J., Foley, A., et al. (2010). Mechanisms of life span extension by rapamycin in the fruit fly Drosophila melanogaster. Cell Metab. 11, 35-46. doi: 10.1016/j.cmet.2009.11.010

Braun, T. P., and Marks, D. L. (2015). The regulation of muscle mass by endogenous glucocorticoids. Front. Physiol. 6:12. doi: 10.3389/fphys.2015.00012

Castets, P., Lin, S., Rion, N., Di Fulvio, S., Romanino, K., Guridi, M., et al. (2013). Sustained activation of mTORC1 in skeletal muscle inhibits constitutive and starvation-induced autophagy and causes a severe, late-onset myopathy. Cell Metab. 17, 731-744. doi: 10.1016/j.cmet.2013.03.015

Chale-Rush, A., Morris, E. P., Kendall, T. L., Brooks, N. E., and Fielding, R. A. (2009). Effects of chronic overload on muscle hypertrophy and mTOR signaling in young adult and aged rats. J. Gerontol. A Biol. Sci. Med. Sci. 64, 1232-1239. doi: 10.1093/gerona/glp146

Coleman, M. E., DeMayo, F., Yin, K. C., Lee, H. M., Geske, R., Montgomery, C., et al. (1995). Myogenic vector expression of insulin-like growth factor I stimulates muscle cell differentiation and myofiber hypertrophy in transgenic mice. J. Biol. Chem. 270, 12109-12116. doi: 10.1074/jbc.270.20.12109

Cuthbertson, D., Smith, K., Babraj, J., Leese, G., Waddell, T., Atherton, P., et al. (2005). Anabolic signaling deficits underlie amino acid resistance of wasting, aging muscle. FASEB J. 19, 422-424. doi: 10.1096/fj.04-2640fje

Deane, C. S., Hughes, D. C., Sculthorpe, N., Lewis, M. P., Stewart, C. E., and Sharples, A. P. (2013). Impaired hypertrophy in myoblasts is improved with testosterone administration. J. Steroid Biochem. Mol. Biol. 138, 152-161. doi: 10.1016/j.jsbmb.2013.05.005

DeYoung, M. P., Horak, P., Sofer, A., Sgroi, D., and Ellisen, L. W. (2008). Hypoxia regulates TSC1/2-mTOR signaling and tumor suppression through REDD1mediated 14-3-3 shuttling. Genes Dev. 22, 239-251. doi: 10.1101/gad.1617608

Dibble, C. C., and Manning, B. D. (2013). Signal integration by mTORC1 coordinates nutrient input with biosynthetic output. Nat. Cell Biol. 15, 555-564. doi: $10.1038 / \mathrm{ncb} 2763$

Drummond, M. J., Dreyer, H. C., Pennings, B., Fry, C. S., Dhanani, S., Dillon, E. L., et al. (2008). Skeletal muscle protein anabolic response to resistance exercise and essential amino acids is delayed with aging. J. Appl. Physiol. 104, 1452-1461. doi: 10.1152/japplphysiol.00021.2008

Fang, Y., Vilella-Bach, M., Bachmann, R., Flanigan, A., and Chen, J. (2001). Phosphatidic acid-mediated mitogenic activation of mTOR signaling. Science 294, 1942-1945. doi: 10.1126/science. 1066015

Florini, J. R., Ewton, D. Z., and Roof, S. L. (1991). Insulin-like growth factor-I stimulates terminal myogenic differentiation by induction of myogenin gene expression. Mol. Endocrinol. 5, 718-724. doi: 10.1210/mend-5-5-718

Fry, C. S., Drummond, M. J., Glynn, E. L., Dickinson, J. M., Gundermann, D. M., Timmerman, K. L., et al. (2011). Aging impairs contraction-induced human skeletal muscle mTORC1 signaling and protein synthesis. Skelet. Muscle 1:11. doi: 10.1186/2044-5040-1-11

Ge, Y., Sun, Y., and Chen, J. (2011). IGF-II is regulated by microRNA-125b in skeletal myogenesis. J. Cell Biol. 192, 69-81. doi: 10.1083/jcb.201007165

Glass, D. J. (2003). Molecular mechanisms modulating muscle mass. Trends Mol. Med. 9, 344-350. doi: 10.1016/S1471-4914(03)00138-2

Glass, D. J. (2005). Skeletal muscle hypertrophy and atrophy signaling pathways. Int. J. Biochem. Cell Biol. 37, 1974-1984. doi: 10.1016/j.biocel.2005.04.018

Gong, Z., Kennedy, O., Sun, H., Wu, Y., Williams, G. A., Klein, L., et al. (2014). Reductions in serum IGF-1 during aging impair health span. Aging Cell 13, 408-418. doi: 10.1111/acel.12188

Guertin, D. A., Stevens, D. M., Thoreen, C. C., Burds, A. A., Kalaany, N. Y., Moffat, J., et al. (2006). Ablation in mice of the mTORC components raptor, rictor, or mLST8 reveals that $\mathrm{mTORC2}$ is required for signaling to Akt-FOXO and PKCalpha, but not S6K1. Dev. Cell 11, 859-871. doi: 10.1016/j.devcel.2006.10.007

Hornberger, T. A. (2011). Mechanotransduction and the regulation of mTORC1 signaling in skeletal muscle. Int. J. Biochem. Cell Biol. 43, 1267-1276. doi: 10.1016/j.biocel.2011.05.007
Hornberger, T. A., Chu, W. K., Mak, Y. W., Hsiung, J. W., Huang, S. A., and Chien, S. (2006). The role of phospholipase $\mathrm{D}$ and phosphatidic acid in the mechanical activation of mTOR signaling in skeletal muscle. Proc. Natl. Acad. Sci. U.S.A. 103, 4741-4746. doi: 10.1073/pnas.0600678103

Hornberger, T. A., Stuppard, R., Conley, K. E., Fedele, M. J., Fiorotto, M. L., Chin, E. R., et al. (2004). Mechanical stimuli regulate rapamycin-sensitive signalling by a phosphoinositide 3-kinase-, protein kinase B- and growth factor-independent mechanism. Biochem. J. 380, 795-804. doi: 10.1042/bj200 40274

Huang, J., and Manning, B. D. (2008). The TSC1-TSC2 complex: a molecular switchboard controlling cell growth. Biochem. J. 412, 179-190. doi: 10.1042/BJ20080281

Izumiya, Y., Hopkins, T., Morris, C., Sato, K., Zeng, L., Viereck, J., et al. (2008). Fast/Glycolytic muscle fiber growth reduces fat mass and improves metabolic parameters in obese mice. Cell Metab. 7, 159-172. doi: 10.1016/j.cmet.2007.11.003

Jacobs, B. L., You, J. S., Frey, J. W., Goodman, C. A., Gundermann, D. M., and Hornberger, T. A. (2013). Eccentric contractions increase the phosphorylation of tuberous sclerosis complex-2 (TSC2) and alter the targeting of TSC2 and the mechanistic target of rapamycin to the lysosome. J. Physiol. 591, 4611-4620. doi: 10.1113/jphysiol.2013.256339

Johnson, S. C., Martin, G. M., Rabinovitch, P. S., and Kaeberlein, M. (2013a). Preserving youth: does rapamycin deliver? Sci. Transl. Med. 5:211fs40. doi: 10.1126/scitranslmed.3007316

Johnson, S. C., Rabinovitch, P. S., and Kaeberlein, M. (2013b). mTOR is a key modulator of ageing and age-related disease. Nature 493, 338-345. doi: 10.1038/nature11861

Korolchuk, V. I., Saiki, S., Lichtenberg, M., Siddiqi, F. H., Roberts, E. A., Imarisio, S., et al. (2011). Lysosomal positioning coordinates cellular nutrient responses. Nat. Cell Biol. 13, 453-460. doi: 10.1038/ncb2204

Laplante, M., and Sabatini, D. M. (2012). mTOR signaling in growth control and disease. Cell 149, 274-293. doi: 10.1016/j.cell.2012.03.017

Lee, S. J. (2007). Quadrupling muscle mass in mice by targeting TGF-beta signaling pathways. PLoS ONE 2:e789. doi: 10.1371/journal.pone.0000789

Leger, B., Derave, W., De Bock, K., Hespel, P., and Russell, A. P. (2008). Human sarcopenia reveals an increase in SOCS-3 and myostatin and a reduced efficiency of Akt phosphorylation. Rejuvenat. Res. 11, 163B-175B. doi: 10.1089/rej.2007.0588

Li, M., Verdijk, L. B., Sakamoto, K., Ely, B., van Loon, L. J., and Musi, N. (2012). Reduced AMPK-ACC and mTOR signaling in muscle from older men, and effect of resistance exercise. Mech. Ageing Dev. 133, 655-664. doi: 10.1016/j.mad.2012.09.001

Lipina, C., Kendall, H., McPherron, A. C., Taylor, P. M., and Hundal, H. S. (2010). Mechanisms involved in the enhancement of mammalian target of rapamycin signalling and hypertrophy in skeletal muscle of myostatin-deficient mice. FEBS Lett. 584, 2403-2408. doi: 10.1016/j.febslet.2010.04.039

Lokireddy, S., Mouly, V., Butler-Browne, G., Gluckman, P. D., Sharma, M., Kambadur, R., et al. (2011). Myostatin promotes the wasting of human myoblast cultures through promoting ubiquitin-proteasome pathwaymediated loss of sarcomeric proteins. Am. J. Physiol. Cell Physiol. 301, C1316C1324. doi: 10.1152/ajpcell.00114.2011

Ma, K., Mallidis, C., Artaza, J., Taylor, W., Gonzalez-Cadavid, N., and Bhasin, S. (2001). Characterization of 5'-regulatory region of human myostatin gene: regulation by dexamethasone in vitro. Am. J. Physiol. Endocrinol. Metab. 281, E1128-E1136.

Ma, K., Mallidis, C., Bhasin, S., Mahabadi, V., Artaza, J., Gonzalez-Cadavid, N., et al. (2003). Glucocorticoid-induced skeletal muscle atrophy is associated with upregulation of myostatin gene expression. Am. J. Physiol. Endocrinol. Metab. 285, E363-E371. doi: 10.1152/ajpendo.00487.2002

Ma, X. M., and Blenis, J. (2009). Molecular mechanisms of mTORmediated translational control. Nat. Rev. Mol. Cell Biol. 10, 307-318. doi: $10.1038 / \mathrm{nrm} 2672$

Manning, B. D., and Cantley, L. C. (2007). AKT/PKB signaling: navigating downstream. Cell 129, 1261-1274. doi: 10.1016/j.cell.2007.06.009

Markofski, M. M., Dickinson, J. M., Drummond, M. J., Fry, C. S., Fujita, S., Gundermann, D. M., et al. (2015). Effect of age on basal muscle protein synthesis and mTORC1 signaling in a large cohort of young and older men and women. Exp. Gerontol. 65, 1-7. doi: 10.1016/j.exger.2015.02.015 
Matsumoto, A., Pasut, A., Matsumoto, M., Yamashita, R., Fung, J., Monteleone, E., et al. (2017). mTORC1 and muscle regeneration are regulated by the LINC00961-encoded SPAR polypeptide. Nature 541, 228-232. doi: 10.1038 /nature21034

McCarthy, J. J., and Esser, K. A. (2010). Anabolic and catabolic pathways regulating skeletal muscle mass. Curr. Opin. Clin. Nutr. Metab. Care 13, 230-235. doi: 10. 1097/MCO.0b013e32833781b5

McFarlane, C., Plummer, E., Thomas, M., Hennebry, A., Ashby, M., Ling, N., et al. (2006). Myostatin induces cachexia by activating the ubiquitin proteolytic system through an NF-kappaB-independent, FoxO1-dependent mechanism. J. Cell. Physiol. 209, 501-514. doi: 10.1002/jcp.20757

McPherron, A. C., Lawler, A. M., and Lee, S. J. (1997). Regulation of skeletal muscle mass in mice by a new TGF-beta superfamily member. Nature $387,83-90$. doi: $10.1038 / 387083 \mathrm{a} 0$

Meijsing, S. H., Pufall, M. A., So, A. Y., Bates, D. L., Chen, L., and Yamamoto, K. R. (2009). DNA binding site sequence directs glucocorticoid receptor structure and activity. Science 324, 407-410. doi: 10.1126/science.1164265

Menconi, M., Fareed, M., O’Neal, P., Poylin, V., Wei, W., and Hasselgren, P. O. (2007). Role of glucocorticoids in the molecular regulation of muscle wasting. Crit. Care Med. 35, S602-S608. doi: 10.1097/01.CCM.0000279194.11328.77

Menon, S., Dibble, C. C., Talbott, G., Hoxhaj, G., Valvezan, A. J., Takahashi, H., et al. (2014). Spatial control of the TSC complex integrates insulin and nutrient regulation of mTORC1 at the lysosome. Cell 156, 771-785. doi: 10.1016/j.cell.2013.11.049

Munck, A., Guyre, P. M., and Holbrook, N. J. (1984). Physiological functions of glucocorticoids in stress and their relation to pharmacological actions. Endocr. Rev. 5, 25-44. doi: 10.1210/edrv-5-1-25

Musaro, A., McCullagh, K., Paul, A., Houghton, L., Dobrowolny, G., Molinaro, M., et al. (2001). Localized Igf-1 transgene expression sustains hypertrophy and regeneration in senescent skeletal muscle. Nat. Genet. 27, 195-200. doi: $10.1038 / 84839$

O'Neil, T. K., Duffy, L. R., Frey, J. W., and Hornberger, T. A. (2009). The role of phosphoinositide 3-kinase and phosphatidic acid in the regulation of mammalian target of rapamycin following eccentric contractions. J. Physiol. 587, 3691-3701. doi: 10.1113/jphysiol.2009.173609

Pallafacchina, G., Calabria, E., Serrano, A. L., Kalhovde, J. M., and Schiaffino, S. (2002). A protein kinase B-dependent and rapamycin-sensitive pathway controls skeletal muscle growth but not fiber type specification. Proc. Natl. Acad. Sci. U.S.A. 99, 9213-9218. doi: 10.1073/pnas.142166599

Parkington, J. D., LeBrasseur, N. K., Siebert, A. P., and Fielding, R. A. (1985). Contraction-mediated mTOR, p70S6k, and ERK1/2 phosphorylation in aged skeletal muscle. J. Appl. Physiol. 97, 243-248.

Passtoors, W. M., Beekman, M., Deelen, J., van der Breggen, Maier, A. B., Guigas, B., et al. (2013). Gene expression analysis of mTOR pathway: association with human longevity. Aging Cell 12, 24-31. doi: 10.1111/acel.12015

Pelosi, L., Giacinti, C., Nardis, C., Borsellino, G., Rizzuto, E., Nicoletti, C., et al. (2007). Local expression of IGF-1 accelerates muscle regeneration by rapidly modulating inflammatory cytokines and chemokines. FASEB J. 21, 1393-1402. doi: 10.1096/fj.06-7690com

Powers, R. W. III., Kaeberlein, M., Caldwell, S. D., Kennedy, B. K., and Fields, S. (2006). Extension of chronological life span in yeast by decreased TOR pathway signaling. Genes Dev. 20, 174-184. doi: 10.1101/gad.1381406

Rabinovsky, E. D., Gelir, E., Gelir, S., Lui, H., Kattash, M., DeMayo, F. J., et al. (2003). Targeted expression of IGF-1 transgene to skeletal muscle accelerates muscle and motor neuron regeneration. FASEB J. 17 53-55. doi: 10.1096/fj.02-0183fje

Ramos, F. J., Chen, S. C., Garelick, M. G., Dai, D. F., Liao, C. Y., Schreiber, K. H., et al. (2012). Rapamycin reverses elevated mTORC1 signaling in lamin A/C-deficient mice, rescues cardiac and skeletal muscle function, and extends survival. Sci. Transl. Med. 4:144ra103. doi: 10.1126/scitranslmed.3003802

Risson, V., Mazelin, L., Roceri, M., Sanchez, H., Moncollin, V., Corneloup, C., et al. (2009). Muscle inactivation of mTOR causes metabolic and dystrophin defects leading to severe myopathy. J. Cell Biol. 187, 859-874. doi: $10.1083 /$ jcb. 200903131

Robida-Stubbs, S., Glover-Cutter, K., Lamming, D. W., Mizunuma, M., Narasimhan, S. D., Neumann-Haefelin, E., et al. (2012). TOR signaling and rapamycin influence longevity by regulating SKN-1/Nrf and DAF-16/FoxO. Cell Metab. 15, 713-724. doi: 10.1016/j.cmet.2012.04.007
Rommel, C., Clarke, B. A., Zimmermann, S., Nunez, L., Rossman, R., Reid, K., et al. (1999). Differentiation stage-specific inhibition of the Raf-MEK-ERK pathway by Akt. Science 286, 1738-1741. doi: 10.1126/science.286.5445.1738

Sakuma, K., Aoi, W., and Yamaguchi, A. (2014). The intriguing regulators of muscle mass in sarcopenia and muscular dystrophy. Front. Aging Neurosci. 6:230. doi: $10.3389 /$ fnagi.2014.00230

Sancak, Y., Bar-Peled, L., Zoncu, R., Markhard, A. L., Nada, S., and Sabatini, D. M. (2010). Ragulator-Rag complex targets mTORC1 to the lysosomal surface and is necessary for its activation by amino acids. Cell 141, 290-303. doi: 10.1016/j.cell.2010.02.024

Sancak, Y., Peterson, T. R., Shaul, Y. D., Lindquist, R. A., Thoreen, C. C., Bar-Peled, L., et al. (2008). The Rag GTPases bind raptor and mediate amino acid signaling to mTORC1. Science 320, 1496-1501. doi: 10.1126/science.1157535

Sandri, M., Barberi, L., Bijlsma, A. Y., Blaauw, B., Dyar, K. A., Milan, G., et al. (2013). Signalling pathways regulating muscle mass in ageing skeletal muscle: the role of the IGF1-Akt-mTOR-FoxO pathway. Biogerontology 14, 303-323. doi: 10.1007/s10522-013-9432-9

Sarbassov, D. D., Ali, S. M., and Sabatini, D. M. (2005). Growing roles for the mTOR pathway. Curr. Opin. Cell Biol. 17, 596-603. doi: $10.1016 /$ j.ceb.2005.09.009

Sartori, R., Gregorevic, P., and Sandri, M. (2014). TGFbeta and BMP signaling in skeletal muscle: potential significance for muscle-related disease. Trends Endocrinol. Metab. 25, 464-471. doi: 10.1016/j.tem.2014.06.002

Sartori, R., Milan, G., Patron, M., Mammucari, C., Blaauw, B., Abraham, R., et al. (2009). Smad2 and 3 transcription factors control muscle mass in adulthood. Am. J. Physiol. Cell Physiol. 296, C1248-C1257. doi: 10.1152/ajpcell.00104.2009

Saxton, R. A., and Sabatini, D. M. (2017). mTOR signaling in growth, metabolism, and disease. Cell 169, 361-371. doi: 10.1016/j.cell.2017. 02.004

Schakman, O., Gilson, H., and Thissen, J. P. (2008). Mechanisms of glucocorticoidinduced myopathy. J. Endocrinol. 197, 1-10. doi: 10.1677/JOE-07-0606

Schiaffino, S., and Mammucari, C. (2011). Regulation of skeletal muscle growth by the IGF1-Akt/PKB pathway: insights from genetic models. Skelet. Muscle 1:4. doi: 10.1186/2044-5040-1-4

Sharples, A. P., Al-Shanti, N., Hughes, D. C., Lewis, M. P., and Stewart, C. E. (2013). The role of insulin-like-growth factor binding protein 2 (IGFBP2) and phosphatase and tensin homologue (PTEN) in the regulation of myoblast differentiation and hypertrophy. Growth Horm. IGF Res. 23, 53-61. doi: 10.1016/j.ghir.2013.03.004

Shimizu, N., Yoshikawa, N., Ito, N., Maruyama, T., Suzuki, Y., Takeda, S., et al. (2011). Crosstalk between glucocorticoid receptor and nutritional sensor mTOR in skeletal muscle. Cell Metab. 13, 170-182. doi: 10.1016/j.cmet.2011.01.001

Song, Z., Moore, D. R., Hodson, N., Ward, C., Dent, J. R., O’Leary, M. F., et al. (2017). Resistance exercise initiates mechanistic target of rapamycin (mTOR) translocation and protein complex co-localisation in human skeletal muscle. Sci. Rep. 7:5028. doi: 10.1038/s41598-017-05483-x

Spangenburg, E. E., Le Roith, D., Ward, C. W., and Bodine, S. C. (2008). A functional insulin-like growth factor receptor is not necessary for load-induced skeletal muscle hypertrophy. J. Physiol. 586, 283-291. doi: 10.1113/jphysiol.2007.141507

Sun, Y., Ge, Y., Drnevich, J., Zhao, Y., Band, M., and Chen, J. (2010). Mammalian target of rapamycin regulates miRNA-1 and follistatin in skeletal myogenesis. J. Cell Biol. 189, 1157-1169. doi: 10.1083/jcb.200912093

Timmerman, K. L., Lee, J. L., Fujita, S., Dhanani, S., Dreyer, H. C., Fry, C. S., et al. (2010). Pharmacological vasodilation improves insulin-stimulated muscle protein anabolism but not glucose utilization in older adults. Diabetes 59, 2764-2771. doi: $10.2337 / \mathrm{db} 10-0415$

Trendelenburg, A. U., Meyer, A., Rohner, D., Boyle, J., Hatakeyama, S., and Glass, D. J. (2009). Myostatin reduces Akt/TORC1/p70S6K signaling, inhibiting myoblast differentiation and myotube size. Am. J. Physiol. Cell Physiol. 296, C1258-C1270. doi: 10.1152/ajpcell.00105.2009

Um, S. H., Frigerio, F., Watanabe, M., Picard, F., Joaquin, M., Sticker, M., et al. (2004). Absence of S6K1 protects against age- and diet-induced obesity while enhancing insulin sensitivity. Nature 431, 200-205. doi: 10.1038/nature02866

Vandenburgh, H. H., Karlisch, P., Shansky, J., and Feldstein, R. (1991). Insulin and IGF-I induce pronounced hypertrophy of skeletal myofibers in tissue culture. Am. J. Physiol. 260, C475-C484. 
Walston, J. D. (2012). Sarcopenia in older adults. Curr. Opin. Rheumatol. 24, 623-627. doi: 10.1097/BOR.0b013e328358d59b

Wang, X., Devaiah, S. P., Zhang, W., and Welti, R. (2006). Signaling functions of phosphatidic acid. Prog. Lipid Res. 45, 250-278. doi: 10.1016/ j.plipres.2006.01.005

Welle, S., Burgess, K., and Mehta, S. (2009). Stimulation of skeletal muscle myofibrillar protein synthesis, p70 S6 kinase phosphorylation, and ribosomal protein S6 phosphorylation by inhibition of myostatin in mature mice. Am. J. Physiol. Endocrinol. Metab. 296, E567-E572. doi: 10.1152/ajpendo.90862.2008

Winbanks, C. E., Weeks, K. L., Thomson, R. E., Sepulveda, P. V., Beyer, C., Qian, H., et al. (2012). Follistatin-mediated skeletal muscle hypertrophy is regulated by Smad3 and mTOR independently of myostatin. J. Cell Biol. 197, 997-1008. doi: $10.1083 /$ jcb.201109091

Xie, Y., Zhang, P., Espinoza, D., Perry, B., Rahnert, J., Zheng, B., et al. (2017). Glucocorticoid-induced inhibition of AKT leads to CREB phosphorylation and increased myostatin expression via a PDE/cAMP/PKA pathway in skeletal muscle. FASEB J. 31:929.922.

Yoon, M. S., Du, G., Backer, J. M., Frohman, M. A., and Chen, J. (2011). Class III PI-3-kinase activates phospholipase D in an amino acid-sensing mTORC1 pathway. J. Cell Biol. 195, 435-447. doi: 10.1083/jcb.201107033

Yoon, M. S., Rosenberger, C. L., Wu, C., Truong, N., Sweedler, J. V., and Chen, J. (2015). Rapid mitogenic regulation of the mTORC1 inhibitor, DEPTOR, by phosphatidic acid. Mol. Cell 58, 549-556. doi: 10.1016/j.molcel.2015.0 3.028

You, J. S., Lincoln, H. C., Kim, C. R., Frey, J. W., Goodman, C. A., Zhong, X. P., et al. (2014). The role of diacylglycerol kinase zeta and phosphatidic acid in the mechanical activation of mammalian target of rapamycin (mTOR) signaling and skeletal muscle hypertrophy. J. Biol. Chem. 289, 1551-1563. doi: 10.1074/jbc.M113.531392

Zhang, Y., Yu, B., He, J., and Chen, D. (2016). From nutrient to MicroRNA: a novel insight into cell signaling involved in skeletal muscle development and disease. Int. J. Biol. Sci. 12, 1247-1261. doi: 10.7150/ijbs. 16463

Conflict of Interest Statement: The authors declare that the research was conducted in the absence of any commercial or financial relationships that could be construed as a potential conflict of interest.

Copyright (c) 2017 Yoon. This is an open-access article distributed under the terms of the Creative Commons Attribution License (CC BY). The use, distribution or reproduction in other forums is permitted, provided the original author(s) or licensor are credited and that the original publication in this journal is cited, in accordance with accepted academic practice. No use, distribution or reproduction is permitted which does not comply with these terms. 